\title{
An Agent-Based Model for Dispatching Real-Time Demand-Responsive Feeder Bus
}

\author{
Xin Li, ${ }^{1}$ Ming Wei, ${ }^{2}$ Jia Hu $\mathbb{D}^{1},{ }^{3}$ Yun Yuan, ${ }^{4}$ and Huifu Jiang ${ }^{5}$ \\ ${ }^{1}$ Department of Electronic Engineering, The Hong Kong Polytechnic University, CF622, Hung Hom, Kowloon, Hong Kong \\ ${ }^{2}$ School of Transportation, Nantong University, No. 9 Seyuan Road, Nantong, Jiangsu Province, China \\ ${ }^{3}$ Key Laboratory of Road and Traffic Engineering of the Ministry of Education, Institute for Advanced Study, \\ Tongji University, Shanghai, China \\ ${ }^{4}$ Department of Civil and Environmental Engineering, University of Wisconsin-Milwaukee, P.O. Box 784, Milwaukee, \\ WI 53201-0784, USA \\ ${ }^{5}$ School of Transportation Science and Engineering, Harbin Institute of Technology, Harbin, China
}

Correspondence should be addressed to Jia Hu; jh8dn@virginia.edu

Received 20 September 2017; Accepted 4 January 2018; Published 26 March 2018

Academic Editor: Yakov Strelniker

Copyright (C) 2018 Xin Li et al. This is an open access article distributed under the Creative Commons Attribution License, which permits unrestricted use, distribution, and reproduction in any medium, provided the original work is properly cited.

\begin{abstract}
This research proposed a feeder bus dispatching tool that reduces rides' effort to reach a feeder bus. The dispatching tool takes in real-time user specific request information and optimizes total cost accordingly (passenger access time cost and transit operation cost) by choosing the best pick-up locations and feeder buses' routes. The pick-up locations are then transmitted back to passengers along with GPS guidance. The tool fits well with the Advanced Traveler Information Services (ATIS) which is one of the six highpriority dynamic mobility application bundles currently being promoted by the United State Department of Transportation. The problem is formulated into a Mixed Integer Programming (MIP) model. For small networks, out-of-the-shelf commercial solvers could be used for finding the optimal solution. For large networks, this research developed a GA-based metaheuristic solver which generates reasonably good solutions in a much shorter time. The proposed tool is evaluated on a real-world network in the vicinity of Jiandingpo metro station in Chongqing, China. The results demonstrated that the proposed ATIS tool reduces both buses operation cost and passenger walking distance. It is also able to significantly bring down computation time from more than 1 hour to about 1 min without sacrificing too much on solution optimality.
\end{abstract}

\section{Introduction}

Over the past decades, rail transit rapidly blooms and benefits the population in both urban and suburban areas. It is widely recognized as a promising mode to shift transport demand to public transport and further enhance urban sustainability [1-4]. However, the difficulty in reaching transit service reduces a great amount of rail transit usage [5-7]. For many potential customers, if it requires driving to reach a rail transit station, they may just drive to their destinations directly. Such predicament is described as the "Last Mile" problem and is a key road block to improving the usage of urban rail transit. A number of solutions have been proposed to better contend with the difficulty. Among them, feeder buses which carry passengers from their own origin of choice to a connecting rail transit station (Figure 1) are one of the most widely accepted solutions [8-11]. The problem associated, which solves for the best feeder bus dispatching plan, is commonly referred to as the Feeder Bus Network Design Problem (FBNDP).

The evolution of consumer access to information about transportation systems in real-time has resulted in a growing number of consumer devices with telecommunications connections. It not only receives transportation information but can also transmit location and user request information. A number of private sector firms have developed devices and applications for collecting, processing, and providing information to end-users about current conditions of transportation systems. This blending of what were considered traditional roles of public and private entities, together 


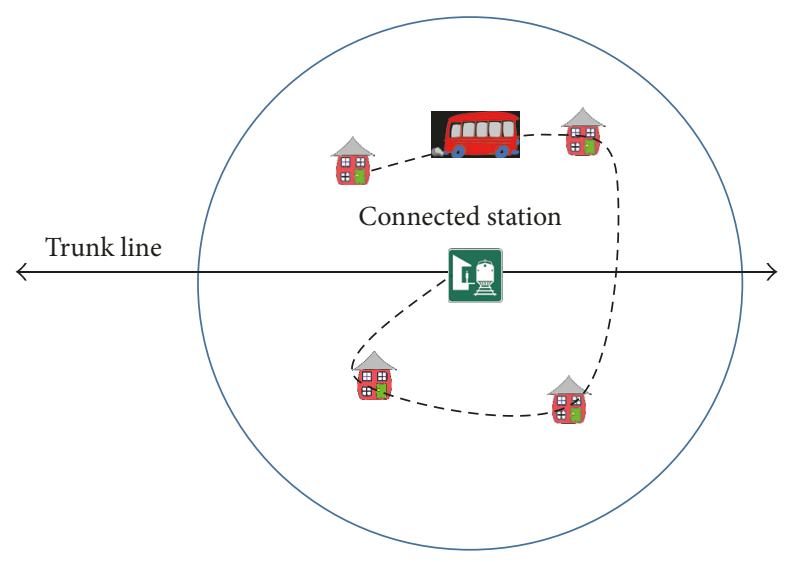

--- DRC

FIGURE 1: Illustration of feeder bus.

with the promise of increased information from Connected Vehicles, provides potentials for a next generation feeder bus dispatching tool that considers real-time information from users, information from vehicles, and information from multiple modes of transportation. This design fits well with the ATIS application which is one of the six highpriority dynamic mobility application bundles promoted by the United State Department of Transportation.

\section{Literature Review}

A number of methodologies have been proposed for solving the FBNDP. In some preliminary designs, route spacing, operating headway, and stop spacing were the major optimization factors [12-15]. In other words, transit route was predetermined without optimization. Hence, enhanced methods were proposed which optimize transit route together with aforementioned factors [16-19]. Although the methods have been enhanced, there are still problems: (1) the access cost from real origin (e.g., home or school) to its corresponding feeder bus stop was ignored when assuming that demands are centered on feeder bus stops (nodes) [17, 18, 20-24]; (2) locations of feeder bus stops are fixed [25, 26]; (3) feeder buses must reach all predetermined stops without considering real-time demand information [11, 18, 27]. They imply a large room for improvement.

Existing solutions to FBNDP can be categorized into the following four categories: mathematical, heuristic, metaheuristic, and hybrid. Mathematical methods are utilized on most simple small toy networks [28, 29]. This kind of approaches is efficient for small size networks [26]. However, when it comes to a large real-world network, there is no guarantee to find an analytic solution [30]. In addition, in most cases, the formulation from a real-world network is NPhard [31] which is not feasible for mathematical methods. To solve these NP-hard problems, heuristic methods were introduced to obtain a near optimum solution [32-34]. These two kinds of solution methods can effectively solve FBNDP for networks under certain size threshold. However, solving time of these two methods grows exponentially with network size [35]. In other words, mathematical methods and heuristic methods lack enough capabilities to afford complexity. Fortunately, with the rapid development of computing power, metaheuristic methods have been developed to handle complicated computation, such as ant colony optimization (ACO) [18], simulated annealing (SA), tabu search (TS) (Fan et al., 2004), and genetic algorithms (GAs) [20, 21, 30, 36]. Compared with heuristic methods, the metaheuristic methods can steadily generate high-quality solutions within an acceptable time frame. However, metaheuristic methods do have one drawback that they are more time consuming for small networks [37]. Hybrid methods can take advantage of different computational techniques [23]. A reasonable combination of the aforementioned solution methods is able to improve the efficiency of metaheuristic methods for small networks. In the past studies, since FBNDP has always been a question at the planning phase, research focus was mostly on solution optimality. In order to prepare for potential real-time applications for ATIS, this paper focuses on the balance of optimality and computation speed. In this paper, it is proposed to use a combination of methods. For small networks, mathematical method is used for better optimality. For large networks, customized GAs is utilized for better efficiency.

2.1. Research Objectives. Given the emerging Advanced Traveler Information Services (ATIS) and shortcoming of the reviewed past studies, this research aims to enhance feeder bus dispatching tool with the following features:

(1) Responsive to real-time traveler information, such as traveler location and destination.

(2) Optimizing not only routes, but also pick-up locations.

(3) Consideration of riders' access cost to pick-up locations.

(4) Accelerated computation speed for potential realtime fleet dispatching.

\section{Methodology}

3.1. Problem Description. This research provides a feeder bus dispatching tool that takes advantage of the Advanced Traveler Information Services (ATIS) features. When in use, passengers submit their locations, number of riders, and desired destinations to the dispatching center using their handhold devices, such as their smart phones. The dispatching center then uses the information to optimize total cost (passenger access time cost and transit operation cost) by choosing the best pick-up locations and feeder bus routing decisions. The pick-up locations are then transmitted back to passengers with GPS guidance.

In this problem, the inputs are passengers' locations, number of riders, and associated desired destination. The objective is to minimize both passenger access to pick-up location time and transit operation cost. The decision factors are pick-up locations and feeder bus routing decisions. 

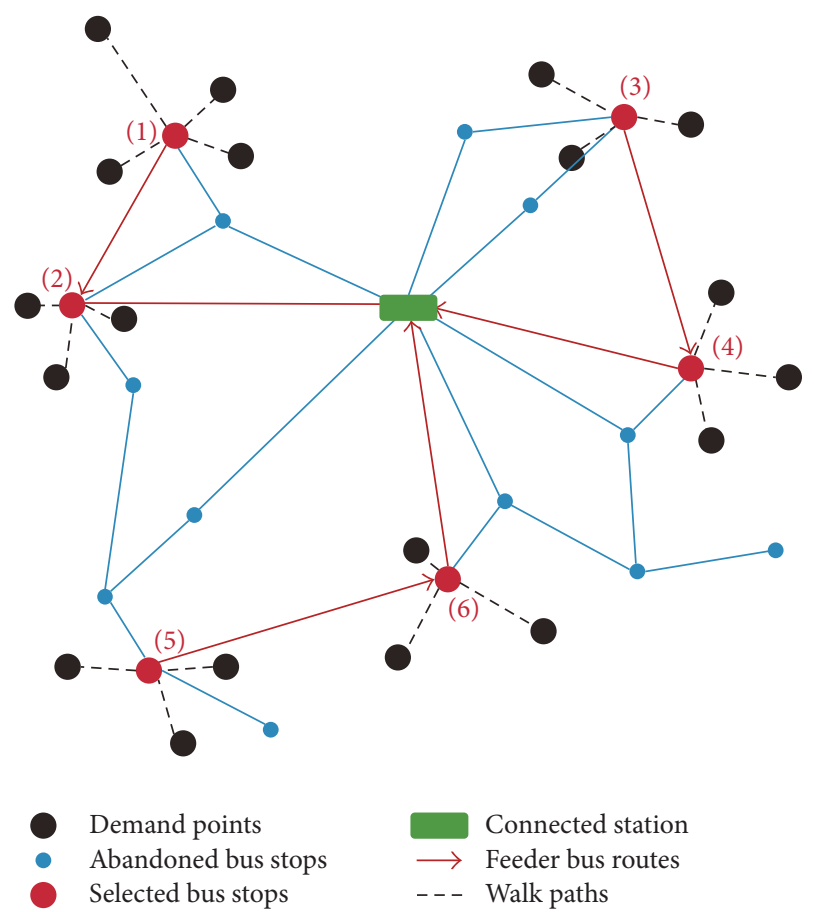

FIGURE 2: Graphical representation of the integrated FBDNP problem.

In this design, although pick-up location is a decision variable in optimization, it is not completely random. To accelerate computation speed, a pool of potential pick-up locations are constructed first. The optimization then chooses the best location from the pool by minimizing total cost to both users and operators. As shown in Figure 2, an illustration of a sample dispatching plan is provided. The black dots are the locations where passenger requests have been submitted. The size of the dots indicates the numbers of passengers at the same location. The blue dots are the potential feeder bus pick-up locations which will not be served in this particular dispatching plan. The red dots are the feeder bus pick-up locations that will be served. Three example bus routes are highlighted in solid red line: (1) node 1-node 2-urban rail transit station; (2) node 3-node 4-urban rail transit station; and (3) node 5-node 6- urban rail transit station.

This research proposed a MILP model with a GA-based metaheuristic solving methodology. It ensures that a realtime response and guidance can be provided to users no matter the size of the network. When the network is small, solvers, such as IBM, ILOG, or CPLEX, can quickly provide the optimal solution. When the network grows exceeding the capacity of the solvers, the proposed GA-based metaheuristic solving methodology can take over and provide near optimal solution in a timely manner. To be noted, the GA algorithm proposed is not simply an out-of-the-shelf commercial software. An additional design is proposed to further improve efficiency and solution quality for potential real-time applications. Detailed information is presented in section "A GA-based Heuristic Algorithm."

\subsection{Model Formulation}

3.2.1. Notation. Notations used hereafter are summarized in "Parameters and Variables in the Mathematical Model" section.

3.2.2. Objective Function. The proposed problem can be formulated as the following Mixed Integer Program (MIP):

Minimize

$$
\begin{aligned}
C_{m} & \sum_{j \in M} \sum_{m \in M \cup M S} \sum_{k \in K} Z_{j m}^{k} d_{j m}{ }^{b} \\
& +C_{h} \sum_{j \in M} \sum_{m \in M \cup M S} \sum_{k \in K} Z_{j m}^{k} t_{j m} \\
& +\sum_{i \in I} \sum_{j \in M} \text { demand }_{i} d_{i j}{ }^{w} x_{i j} .
\end{aligned}
$$

In this formulation, the objective function is given by (1), which includes three terms: the first term is the travel distance of feeder buses, the second term is total in-vehicle time, and the third term represents feeder users' walking distance to pick-up stations. The first and second terms minimize the total operational cost of feeder bus system while the third term minimizes the total walking distances for passengers.

3.2.3. Constraints. The following constraint limits the maximum number of pick-up locations so that the usage of feeder bus is not abused:

$$
\sum_{j \in M} y_{j} \leq P .
$$

The following constraints guarantee that each passenger is assigned to one selected pick-up location and one pick-up location only:

$$
\begin{aligned}
x_{i j} & \leq y_{j} \quad \forall i \in I, \forall j \in M ; \\
\sum_{j \in M} x_{i j} & =1 \quad \forall i \in I .
\end{aligned}
$$

The following constraint prevents bus routing from connecting to an unselected potential pick-up location:

$$
2 * Z_{j m}^{k} \leq y_{j}+y_{m}
$$

$$
\forall k \in K, \forall j \in M, \forall m \in M \cup M S .
$$

The following constraint prevents any selected pick-up location being double served by multiple feeder buses. This constraint further increases efficiency of the entire system:

$$
\sum_{j \in M} \sum_{k \in K} Z_{j m}^{k} \leq 1 ; \quad \forall m \in M
$$

The following constraints ensure that each selected pickup location has the same incoming arc and outgoing arc:

$$
\begin{gathered}
\sum_{p \in M} \sum_{k \in K} Z_{p j}^{k} \leq 1 ; \quad \forall j \in M ; \\
\sum_{m \in M \cup M S} z_{j m}^{k}-\sum_{p \in M} Z_{p j}^{k} \geq 0 ; \quad \forall j \in M, \quad \forall k \in K .
\end{gathered}
$$


The following constraint is used for subtour elimination in vehicle routing problem and is with polynomial cardinality [38]:

$$
U_{i k}-U_{j k}+\left(|\mathscr{M}| * Z_{j m}^{k}\right) \geq|M|-1,
$$

$\forall j, m \in M \cup M S, \forall k \in K$.

The following constraint guarantees the number of passengers boarded never exceeds vehicle capacity during the entire feeder bus trip:

$$
\sum_{j \in M} \sum_{m \in M \cup M S} c_{j m}^{k} \leq Q_{k} ; \quad \forall k \in K .
$$

The following constraints ensure that no passenger is left behind:

$$
\begin{aligned}
\sum_{m \in M \cup M S} \sum_{k \in K} c_{j m}^{k} & =\sum_{i \in I} \operatorname{Demand}_{i} * x_{i j} ; \quad \forall j \in M ; \\
c_{j m}^{k}-Z_{j m}^{k} & \geq 0 ;
\end{aligned}
$$

$$
\forall j \in M, \forall m \in M \cup \mathrm{MS}, \forall k \in K \text {. }
$$

The following constraint guarantees that passengers are assigned to the correct feeder lines:

$$
c_{j m}^{k} \leq Q_{k} * Z_{j m}^{k} ; \quad \forall j \in M, \forall m \in M \cup M S, \forall k \in K .
$$

The following constraints are used to limit the minimum length and maximum travel time for each feeder bus route. This constraint is used to improve travel time reliability:

$$
\begin{gathered}
\sum_{j \in M} \sum_{m \in M \cup M S} Z_{j m}^{k} * t_{j m} \leq T_{\max } ; \quad \forall k \in K ; \\
\sum_{j \in M} \sum_{m \in M \cup M S} Z_{j m}^{k} * d_{j m}{ }^{b} \geq L_{\min } ; \quad \forall k \in K .
\end{gathered}
$$

The following constraints ensure that all feeder bus routes lead to a rail transit station as their final destination:

$$
\begin{aligned}
& \sum_{j \in M} \sum_{m \in \mathrm{MS}} Z_{j m}^{k}=1 ; \quad \forall k \in K ; \\
& \sum_{j \in M} \sum_{m \in \mathrm{MS}} Z_{m j}^{k} \leq 0 ; \quad \forall k \in K .
\end{aligned}
$$

3.3. A GA-Based Heuristic Algorithm. The proposed optimization model is nondeterministic polynomial-time hard (NP-hard). For small-scale networks, solvers, such as IBM, ILOG, or CPLEX, could be applied to find the optimal solution. However, when it comes to large-scale networks, the aforementioned solvers would either fail or compute for a very long time which is not acceptable for a real-time fleet dispatching tool. Thus, a GA-based heuristic approach is further developed to reduce computation time for potential real-time applications. The following provides a detailed description of the proposed GA-based algorithm.
3.3.1. GA Chromosomes Structure. The proposed GA chromosomes consists of three parts: pick-up location selection $\left(y_{j}\right)$, demand assignment $\left(x_{i j}\right)$, and feeder bus routing decision $\left(Z_{j m}^{k}\right)$. The following provides a detailed explanation of each part of the proposed GA chromosomes. Integer vector $U=\left(u_{1}, u_{2}, \ldots, u_{\mathscr{M}}, u_{\mathscr{M}+1}, \ldots, u_{2 \mathscr{M}}, u_{2 \mathscr{M}+1}, \ldots, u_{2 \mathscr{M}+\mathscr{I}}\right)$ is used to represent the solution to the proposed model:

(1) The first part of the proposed GA chromosomes $\left(u_{1}, u_{2}, \ldots, u_{j}, \ldots, u_{\mathscr{M}}\right)$ (a vector of binary variables) represents the decision of pick-up locations selection. If $u_{j}=1$, then the corresponding candidate node $j$ is selected and will be served by a feeder bus;

(2) The second part of the proposed GA chromosomes $\left(u_{\mathscr{U}+1}, u_{\mathscr{M}+2}, \ldots, u_{\mathscr{M}+j}, \ldots, u_{2 \mathscr{M}}\right)$ (a vector of integer variables) is used to assign the selected pick-up locations to each route. $u_{M+j}$ ranges from 1 to $k$, where $k \in K$ is the index of feeder bus. For example, $u_{M+2}=$ 3 indicates that bus stop $j=2$ is assigned to feeder bus number 3. Dijkstra algorithm is used to find the sequence of serving that provides the shortest bus route;

(3) The third part of the proposed GA chromosomes

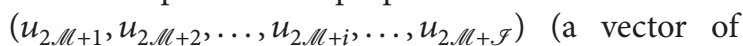
integer variables) is used to assign passengers to selected pick-up locations. $u_{2 M+\mathscr{I}}$ ranges from 1 to $m$, where $m \in M$ is the index of pick-up locations.

3.3.2. Fitness Evaluation. To reinforce constraints (8), (11) in the proposed GA algorithm, corresponding penalty terms are added to the cost function (1) for generating a fitness function:

$$
\begin{aligned}
F & =f+M_{1} \cdot \sum_{k \in K}\left(\max \left(\sum_{j \in M} \sum_{m \in M \cup M S} c_{j m}^{k}-Q_{k}, 0\right)\right)^{2} \\
& +M_{2} \\
& \cdot \sum_{k \in K}\left(\max \left(\sum_{j \in M} \sum_{m \in M \cup M S} Z_{j m}^{k} * t_{j m} \leq T_{\max }, 0\right)\right)^{2} \\
& +M_{3} \\
& \cdot \sum_{k \in K}\left(\max \left(\sum_{j \in M} \sum_{m \in M \cup M S} Z_{j m}^{k} * d_{j m} \geq L_{\min }, 0\right)\right)^{2}
\end{aligned}
$$

where $f$ is the objective function (1) of the aforementioned MILP model; $F$ is the function used in fitness evaluation; $M_{1}, M_{2}, M_{3}$ are large positive penalty constants.

3.3.3. Initial Population Generation. The quality of the solution found and the convergence speed of using a GAbased algorithm, highly depend on the selection of the initial population. In this research, a Heuristic Algorithm to efficiently generate a decent initial population is proposed. The procedures are explained as follows. 


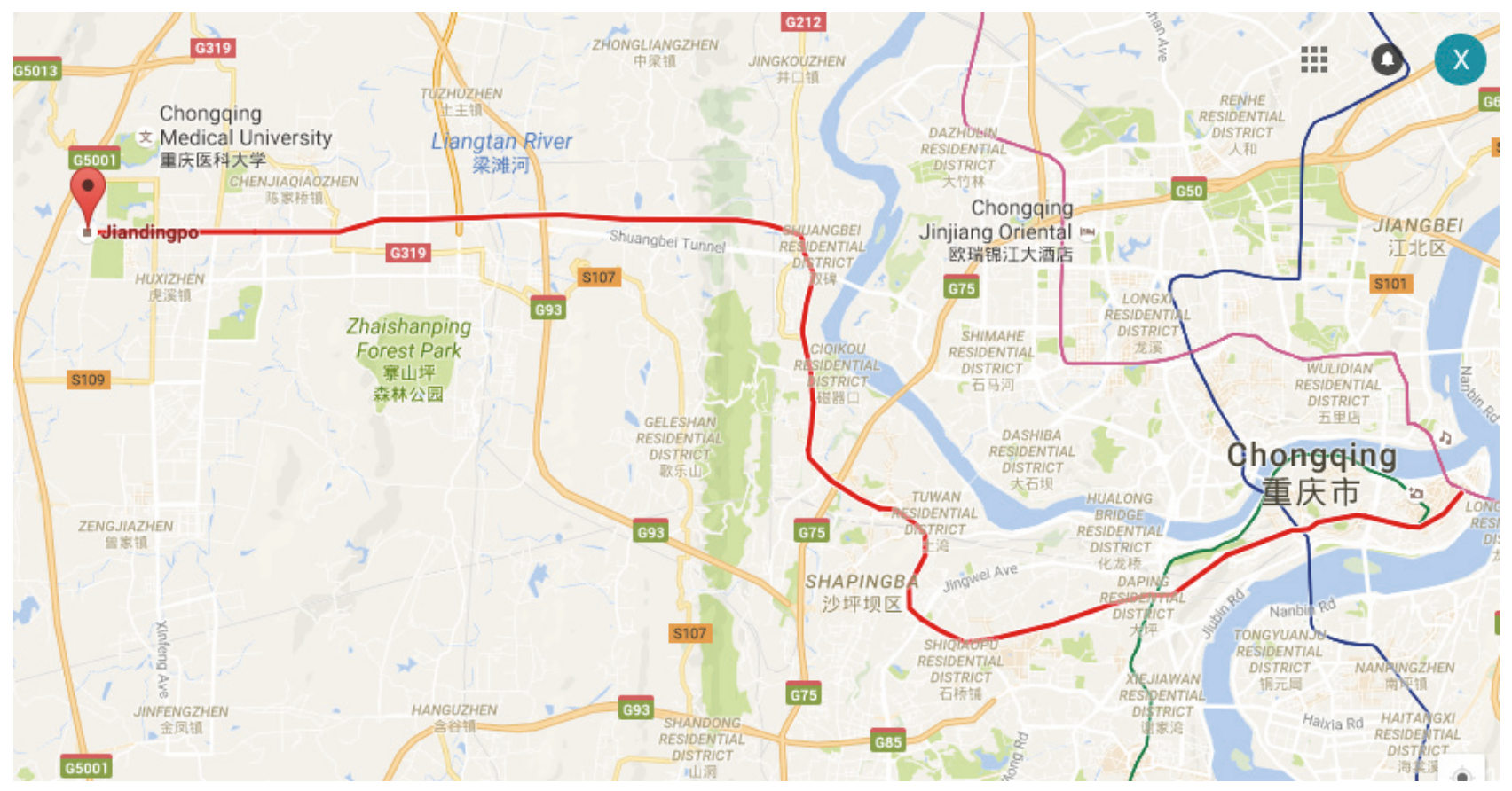

FIgURE 3: Geolocation of Jiandingpo Station (source: Google Map).

Step 1. Define parameters, such as $M$ (the set of candidate pick-up locations), MS (rail transit station), and $K$ (the set of feeder bus routes).

Step 2. Use inverse-search method to generate a feasible routing plan for each feeder bus:

(1) Start from the node of rail transit station;

(2) Initiate a feasible set of candidate bus stops $M^{\prime} \in M$ in which the distance between each selected node and rail station is less than $L_{\min }$;

(3) Randomly pick a candidate pick-up location from $M^{\prime}$ to connect with the node of rail station, and remove this pick-up location node from $M^{\prime}$;

(4) Repeat (3) until the constraints (2) and (11) are violated.

Step 3. Set $y_{j}=y_{m}=1$ for each feeder bus $k \in K$ with $Z_{j m}^{k}=1$, as defined in Step 2.

Step 4. Determine the values of $x_{i j}$.

Step 5. Generate the initial population $U$ with the results of $y_{j}, x_{i j}, z_{j m}^{k}$ from Step 1 to Step 4 .

3.3.4. Genetic Selection Operators. This operation gives preference to better solutions (chromosomes) in order to pass on their good "genes" to the next generation. This study uses both random competition and elitist selection strategies to ensure that "genes" with the highest fitness in the previous population are retained in the next population.
3.3.5. Crossover and Mutation. Crossover operator simulates exchanging and recombining part of genes in two individuals to produce new individuals in an evolutionary process. In this study, one-point method is utilized which randomly selects an integer $P \in[1,(2 \mathscr{M}+\mathscr{I})]$ and exchange the front and the rear portions of the two parents $U_{1}$ and $U_{2}$ to generate new offspring chromosomes $U_{1}^{\prime}$ and $U_{2}^{\prime}$. Mutation operator also follows one-point method where mutation fraction is defined to be 0.15 . If gene $u_{j} \in U$ has been selected as a mutation point, then $u_{j}$ is set to 1 or 0 for all $j \in M$ while $j$ randomly takes value from the set $K$.

3.3.6. Stopping Criteria. The GA stops evolving when the following criteria are met:

(1) $\left|\left(\widehat{\mathscr{F}}_{\text {min }}^{n}-\widehat{\mathscr{F}}_{\text {min }}^{n-1}\right) / \widehat{\mathscr{F}}_{\text {min }}^{n}\right|<\epsilon$; that is, the difference between the minimum evaluation values between two consecutive generations is less than a threshold $\epsilon$; or

(2) A preset maximal number of generations are reached.

\section{Evaluation}

\subsection{Experimental Design}

4.1.1. Network Setup. The proposed feeder bus dispatching tool is tested on a real-world network in the vicinity of Jiandingpo metro station in Chongqing, China. Chongqing is the largest city in the west of China. The Jiandingpo metro station is located in the west of Chongqing which is a low population density area, as demonstrated in Figure 3. The development of feeder system is able to effectively improve the accessibility of local urban rail stations and further increase transit ridership. 
TABLE 1: Real-time number of passengers of each request.

\begin{tabular}{|c|c|}
\hline Demand point & Number of passengers \\
\hline D1 & 23 \\
\hline $\mathrm{D} 2$ & 36 \\
\hline D3 & 50 \\
\hline D4 & 7 \\
\hline D5 & 22 \\
\hline D6 & 70 \\
\hline D7 & 11 \\
\hline D8 & 25 \\
\hline D9 & 32 \\
\hline D10 & 21 \\
\hline D11 & 30 \\
\hline D12 & 17 \\
\hline D13 & 28 \\
\hline D16 & 6 \\
\hline D17 & 12 \\
\hline D18 & 25 \\
\hline D19 & 12 \\
\hline D20 & 8 \\
\hline D21 & 6 \\
\hline $\mathrm{D} 22$ & 7 \\
\hline D23 & 6 \\
\hline $\mathrm{D} 24$ & 5 \\
\hline D25 & 5 \\
\hline D14 & 24 \\
\hline D15 & 25 \\
\hline
\end{tabular}

Key parameters used in the case study are given as below:

(i) Route capacity: 200 persons;

(ii) Maximum allowed number of stops: 16;

(iii) Maximum allowed travel time for each route: 20 mins;

(iv) Minimum route length: $2 \mathrm{~km}$;

(v) Operational cost for feeder buses: $\$ 3$ per km;

(vi) Operational cost for drivers: $\$ 5$ per $\mathrm{h}$;

(vii) A total of 25 ride requests have been received;

(viii) Potential pick-up location number is 42 .

The test was conducted on a real network, but the data is hypothetical. In our study, historical data is generated randomly. "Real-time data" is acquired by adding stochasticity to historical data. As a result, there is discrepancy between historical data and real-time data.

The real-time number of passengers of each request is recorded in Table 1.

The historical number of passengers of each request is recorded in Table 2.

Figure 4 demonstrates the geographic location of all passengers and potential pick-up locations. The red dots represent all passengers. The size of the red dots represents passenger number. The blue dots represent candidate pick-up locations. The green dot is the Jiandingpo metro station.
TABLE 2: Historical number of passengers of each request.

\begin{tabular}{lc}
\hline Demand point & Number of passengers \\
\hline D1 & 10 \\
D2 & 2 \\
D3 & 8 \\
D4 & 7 \\
D5 & 5 \\
D6 & 6 \\
D7 & 8 \\
D8 & 10 \\
D9 & 6 \\
D10 & 8 \\
D11 & 10 \\
D12 & 25 \\
D13 & 15 \\
D16 & 38 \\
D17 & 15 \\
D18 & 18 \\
D19 & 35 \\
D20 & 30 \\
D21 & 18 \\
D22 & 11 \\
D23 & 35 \\
D24 & 25 \\
D25 & 10 \\
D14 & 32 \\
D15 & 21 \\
\hline &
\end{tabular}

4.1.2. Scenarios. A total of three scenarios have been tested in order to show the advantage of having ATIS and the proposed GA-based metaheuristic solver:

(i) Proposed Algorithm: in this scenario, the proposed MILP model is used and solved by the proposed GAbased metaheuristic solver.

(ii) Solver Baseline: in the scenario, the proposed MILP model is still used but solved by out-of-the-shelf commercial solver CPLEX 12.6. This baseline is tested to show the efficiency of the proposed GA-based algorithm.

(iii) ATIS Baseline: in this scenario, the proposed MILP model is still used, but the passenger demand is from historical data instead of real-time. This baseline shows the advantage of having ATIS feature in a feeder bus dispatching tool.

4.1.3. Analysis Methodology. The proposed algorithm is compared against the Solver Baseline and ATIS Baseline to show the benefit of considering real-time passenger request and using the proposed GA-base algorithm. Sensitivity analysis has been conducted in terms of the number of routes provided. Measurements of Effectiveness (MOE) adopted include computation time, average route distance, average on-vehicle travel time, and average weighted walking 


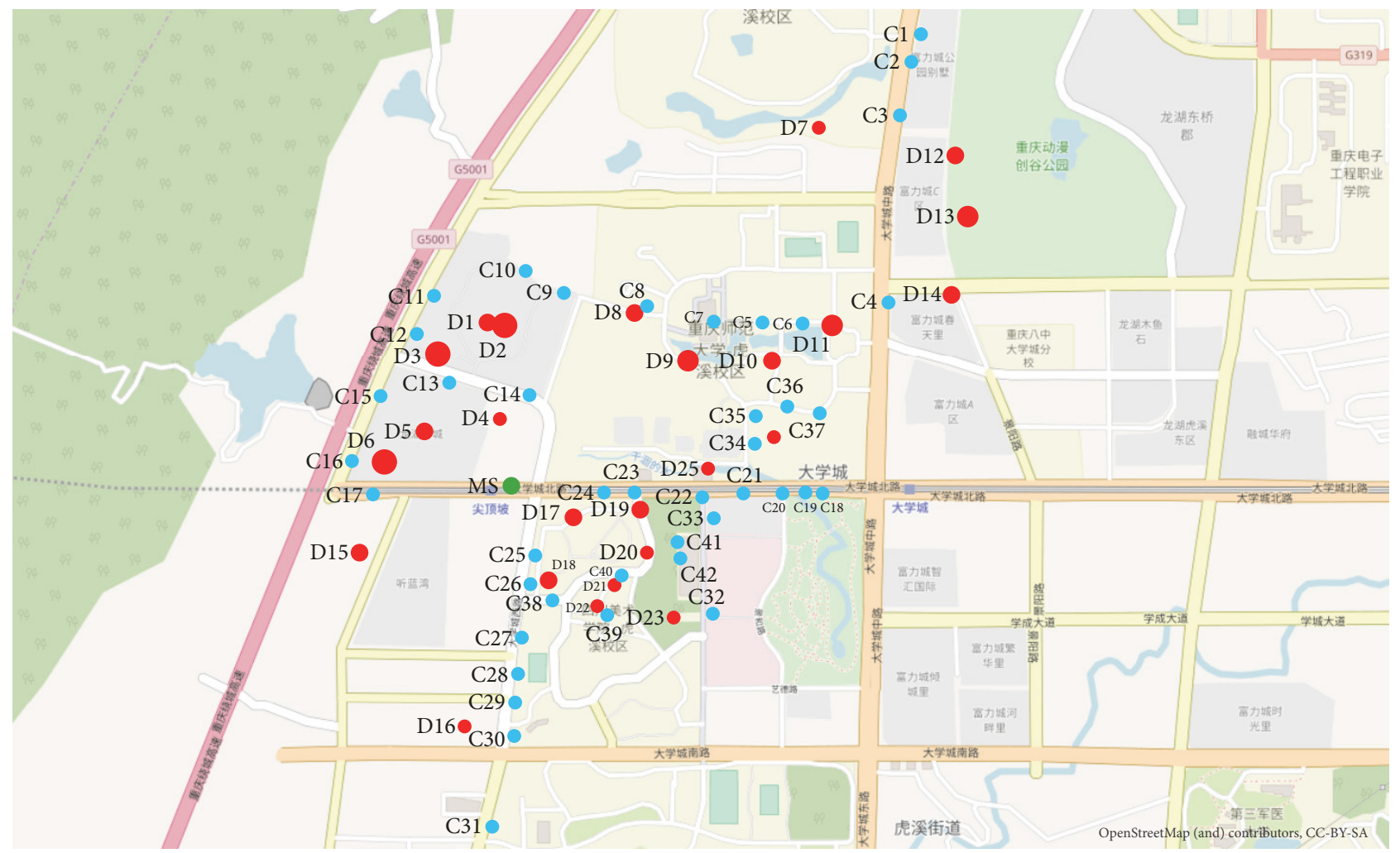

FIGURE 4: Spatial distribution of demand using Jiandingpo Station and candidate bus stops (map resource: Baidu Map).

distance. The model is solved using a computer with Windows 8 , a $2.8 \mathrm{GHz}$ processor, and $8 \mathrm{~GB}$ of RAM.

4.2. Results. The following presents a showcase of sample optimization outputs assuming three feeder bus routes are provided. Figure 5 demonstrates the routing plans for Solver Baseline scenario. The red solid line represents Route 1, the blue solid line represents Route 2, and the black dash line represents Route 3. Green solid lines are used to indicate which pick-up location the passengers are associated with.

Figure 6 demonstrates the routing plans for ATIS Baseline scenario. The legend is the same as Figure 5. The red solid line represents Route 1, the blue solid line represents Route 2, and the black dash line represents Route 3. Green solid lines are used to indicate which pick-up location the passengers are associated with.

Finally, Figure 7 demonstrates the routing plans for the proposed algorithm scenario.

The results from the comparison of the proposed algorithm scenario and the Solver Baseline scenario are presented in Table 3. In short, the proposed algorithm is able to provide reasonably good solutions in a much faster manner. As shown in Table 3, the increase of route number dramatically brings up computation time when using CPLEX. When the number of routes is 5 , it takes more than one hour to generate the optimal solution. This is definitely not acceptable for ATIS which requires real-time response. The proposed algorithm is not sensitive to network complexity and stably generates near optimal solutions in about one minute. The quality of the solutions provided by the proposed algorithm is also reasonably good. It is typically about $10-20 \%$ above the true optimal, while worst case is about $30 \%$ above. This is a necessary trade-off between optimality and efficiency. Figure 8 shows the convergence of the proposed algorithm.

The results from the comparison of the proposed algorithm scenario and the ATIS Baseline scenario are presented in Table 4. Intuitively, with the true real-time passenger request information, instead of using historical average data, the proposed algorithm saves operational cost for bus fleet and reduces riders' walking distances. In the case study tested, feeder bus fleet saves about $12 \%$ of its cost and riders access to the pick-up locations is made easier by about $9 \%$.

\section{Conclusion}

This research proposed a feeder bus dispatching tool that reduces rides' effort to reach a feeder bus. The dispatching tool takes in user specific information and optimizes total cost accordingly (passenger access time cost and transit operation cost) by choosing the best pick-up location and feeder bus routing. The pick-up location is then transmitted back to passengers with GPS guidance. The tool fits well with the Advanced Traveler Information Services (ATIS) which is one of the six high-priority dynamic mobility application bundles currently being promoted by the United State Department of Transportation. The problem is formulated into a Mixed Integer Programming (MIP) model. For small networks, 


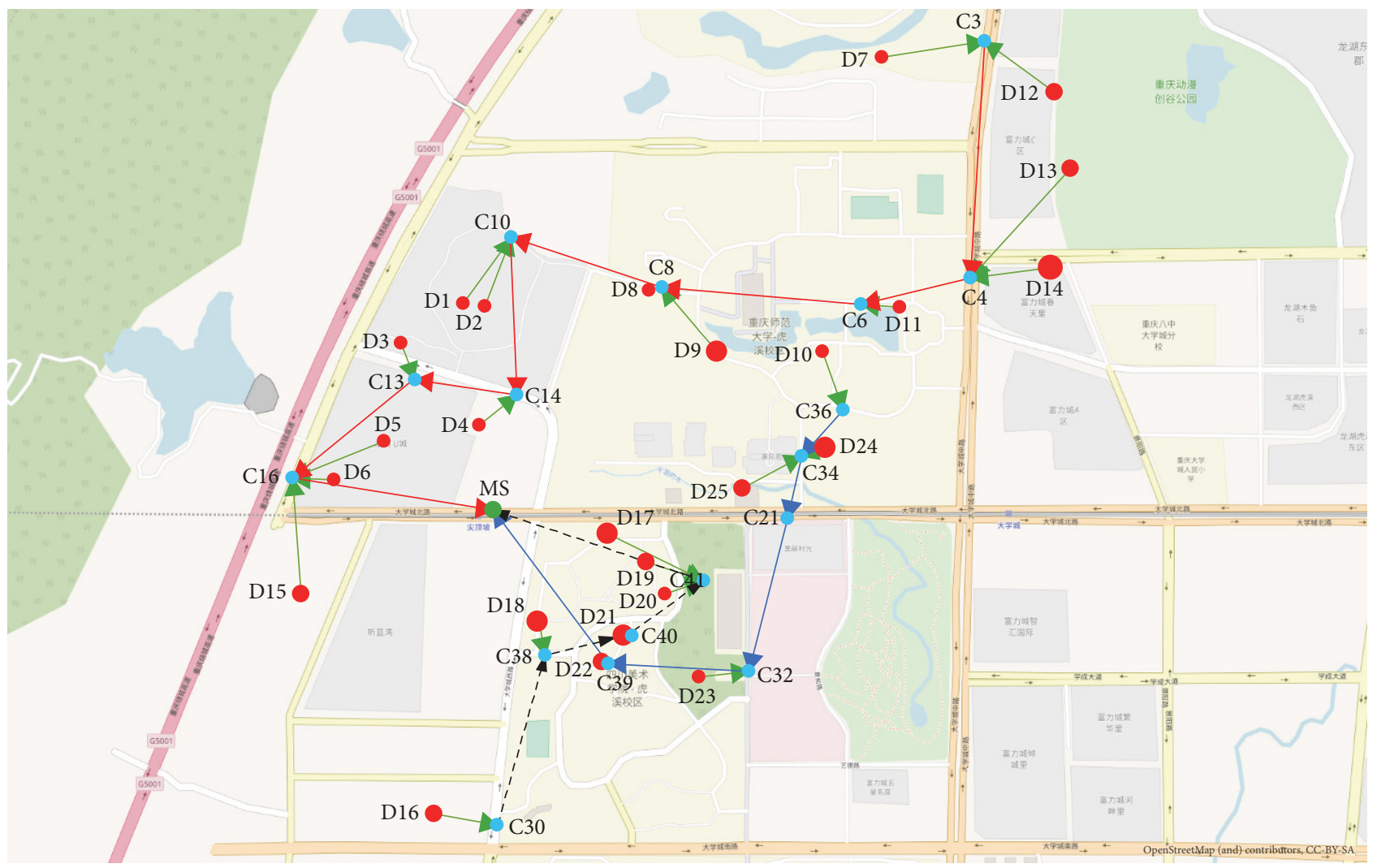

FIGURE 5: Solver baseline result (map source: Google).

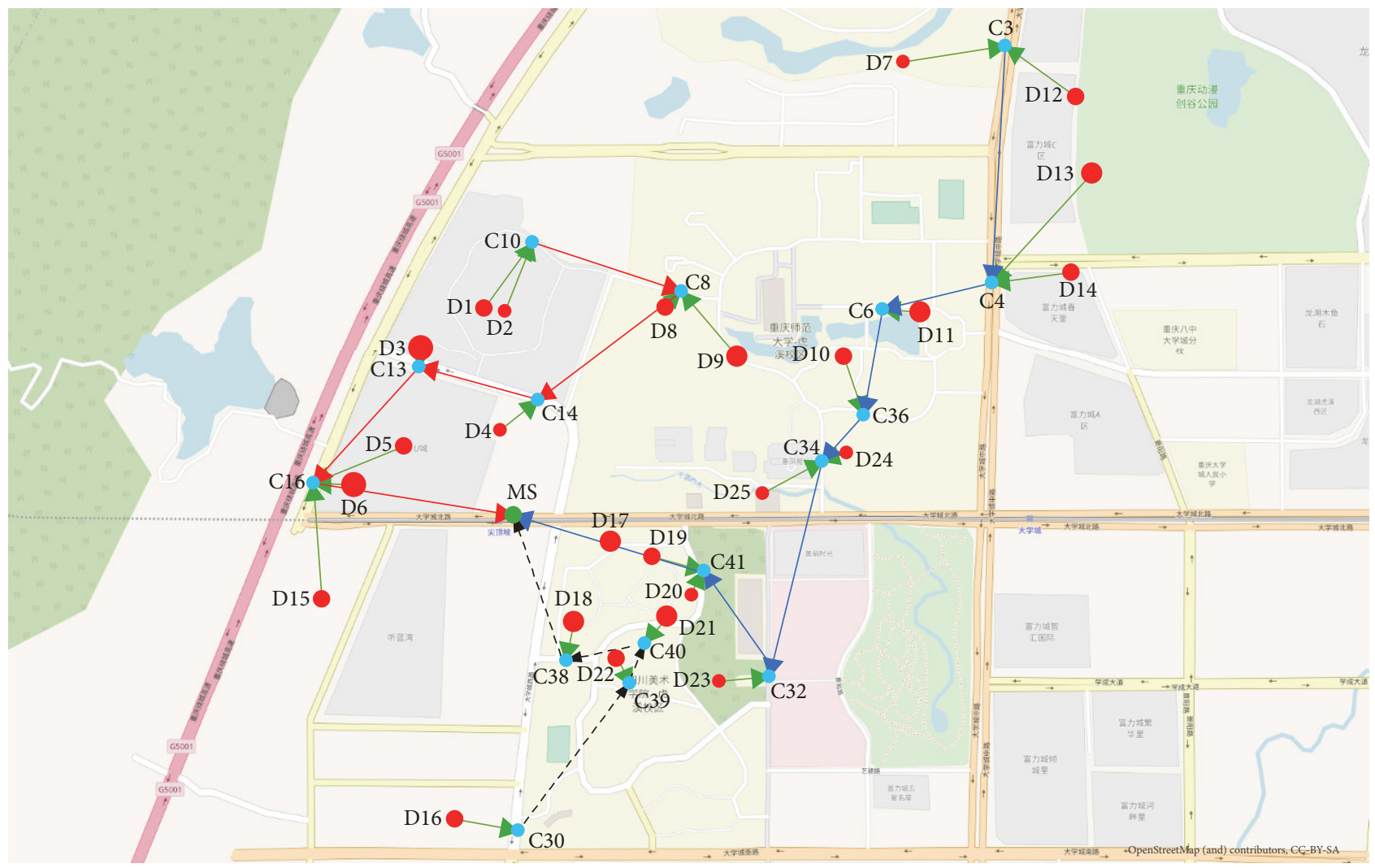

FIGURE 6: ATIS baseline result (map source: Google). 


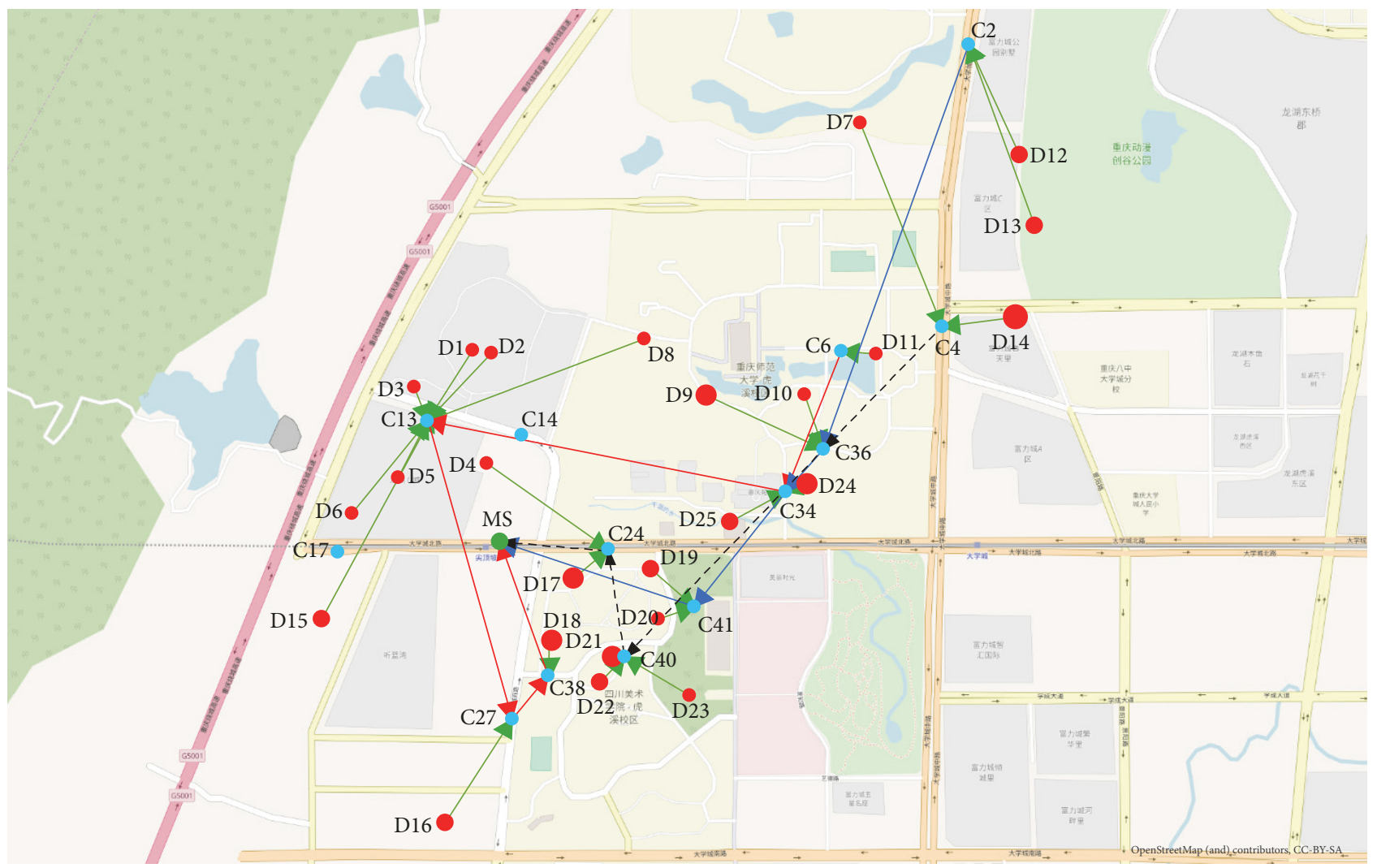

FIgURE 7: Proposed algorithm results (map source: Google).

TABLE 3: Comparison of CPLEX solution and heuristic solution.

\begin{tabular}{|c|c|c|c|c|}
\hline Scenario & Solved time (s) & Average route length $(\mathrm{km})$ & Average travel time (min) & Average weighted walking distance $(\mathrm{m})$ \\
\hline \multicolumn{5}{|c|}{ CPLEX results } \\
\hline 3 routes & 390 & 3.67 & 11 & 253.74 \\
\hline 4 routes & 573 & 2.61 & 7.85 & 253.74 \\
\hline 5 routes & 3813 & 2.29 & 6.72 & 253.74 \\
\hline \multicolumn{5}{|c|}{ Heuristic results } \\
\hline 3 routes & 48 & 4.17 & 12.44 & 292.94 \\
\hline 4 routes & 66 & 3.15 & 9.63 & 307.13 \\
\hline 5 routes & 82 & 2.64 & 7.89 & 329.59 \\
\hline \multicolumn{5}{|l|}{ Difference } \\
\hline 3 routes & $-88 \%$ & $14 \%$ & $13 \%$ & $15 \%$ \\
\hline 4 routes & $-88 \%$ & $21 \%$ & $23 \%$ & $21 \%$ \\
\hline 5 routes & $-98 \%$ & $15 \%$ & $17 \%$ & $30 \%$ \\
\hline
\end{tabular}

TABLE 4: Comparison of with and without ATIS.

\begin{tabular}{lccc}
\hline Scenario & $\begin{array}{c}\text { Average route } \\
\text { length }(\mathrm{km})\end{array}$ & $\begin{array}{c}\text { Average } \\
\text { travel time } \\
(\mathrm{min})\end{array}$ & $\begin{array}{c}\text { Average } \\
\text { weighted } \\
\text { walking } \\
\text { distance }(\mathrm{m})\end{array}$ \\
\hline ATIS baseline & 3.67 & 11.00 & 253.74 \\
Proposed algorithm & 3.21 & 9.84 & 231.72 \\
Savings & $13 \%$ & $11 \%$ & $9 \%$ \\
\hline
\end{tabular}

out-of-the-shelf commercial solver could be used for finding the optimal solution. For large networks, this research developed a GA-based metaheuristic solver which generates a reasonably good solution in a much faster manner. The proposed tool is evaluated on a real-world network in the vicinity of Jiandingpo metro station in Chongqing, China. The results demonstrated that the proposed tool is able to significantly reduce computation time from more than 1 hour down to about $1 \mathrm{~min}$ without sacrificing too much on solution 


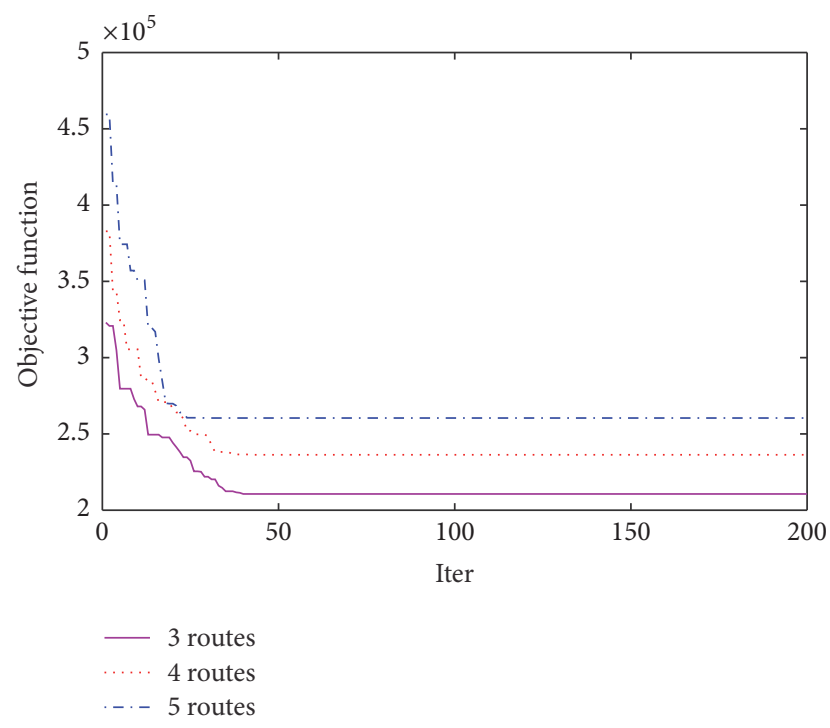

FIGURE 8: Convergence process of GA algorithm of three scenarios.

optimality. Detailed evaluation on the proposed tool reveals the following:

(i) The proposed tool greatly reduces computation time. The computation time is consistently around 1 minute, even for networks that take CPLEX more than 1 hour to solve. It is ready for potential ATIS application.

(ii) The quality of the solutions provided by the proposed algorithm is reasonably good. It is typically about $10-20 \%$ above the true optimal, while the worst case is about $30 \%$ above.

(iii) With the true real-time passenger request information, instead of using historical averaged data, the proposed algorithm saves operational cost for bus fleet and reduces riders walking distances.

Future research could further develop the proposed tool to consider the departure time of riders so that feeder buses' routing determination process could divide passengers into different trips and plan accordingly. Additional efforts could also be applied to further reduce computation time.

\section{Parameters and Variables in the Mathematical Model}

\section{Indices}

$i$ : $\quad$ Passenger location index

$j, m, p$ : Vehicular node (bus stop candidates and urban rail transit station) index

$k$ : $\quad$ Bus route index.

Sets

I: $\quad$ Set of $\mathscr{I} \in \mathbb{R}$ passenger locations

$M$ : Set of $\mathscr{M} \in \mathbb{R}$ potential pick-up locations
MS: Set of urban rail transit stations

$K: \quad$ Set of $\mathscr{K} \in \mathbb{R}$ bus routes.

\section{Parameters}

Demand $_{i}$ : Number of passengers at location $i ; i \in I$

$P$ : $\quad$ Maximum number of designed bus stops

$Q_{k}$ : $\quad$ Capacity of bus route $k ; k \in K$

$T_{\max }: \quad$ Maximum travel time

$L_{\text {min }}: \quad$ Minimum route length

$d_{i j}{ }^{w}$ : $\quad$ Map-based walking distance from demand

point $i$ to pick-up location $j ; i \in I, j \in M$

$d_{\text {jm }}: \quad$ Map-based bus driving distance from

node $j$ to node $m ; j, m \in M \cup M S$

$t_{j m}$ : $\quad$ Map-based bus travel time from node $j$ to node $m ; j, m \in M \cup M S$

$C_{m}: \quad$ Operational cost per km (unit: dollar)

$C_{h}$ : Operational cost for drivers per operating hour (unit: dollar).

\section{Decision Variables}

$c_{j m}^{k}$ : Number of passengers at pick-up location $j$ assigned to route $k$ traveling from $j$ to $m$ (unit: person)

$U_{i k}$ : An auxiliary (real) variable for subtour elimination constraint in route of bus $k$

$Z_{j m}^{k}: Z_{j m}^{k}=1$, if pick-up location $j$ precedes pick-up location $m$ on the route $k ; Z_{j m}^{k}=0$, otherwise

$x_{i j}: \quad x_{i j}=1$, if passenger location $i$ is assigned to pick-up location $j ; x_{i j}=0$, otherwise

$y_{j}: \quad y_{j}=1$, if candidate node $j$ is selected as a pick-up location; $y_{j}=0$, otherwise.

\section{Conflicts of Interest}

The authors declare that they have no conflicts of interest.

\section{Acknowledgments}

This research is supported by the 13th Five-Year plan of Jiangsu Open university (no. 16SSW-Z-002).

\section{References}

[1] A. T. Murray, R. Davis, R. J. Stimson, and L. Ferreira, "Public transportation access," Transportation Research Part D: Transport and Environment, vol. 3, no. 5, pp. 319-328, 1998.

[2] A. Verma, D. Upadhyay, and R. Goel, "An integrated approach for optimal rail transit corridor identification and scheduling using geographical information system," Journal of King Saud University - Science, vol. 23, no. 3, pp. 255-271, 2011.

[3] L. He, Q. Liang, and S. Fang, "Challenges and innovative solutions in urban rail transit network operations and management: China's Guangzhou metro experience," Urban Rail Transit, vol. 2, no. 1, pp. 33-35, 2016.

[4] Ø. Engebretsen, P. Christiansen, and A. Strand, "Bergen light rail - Effects on travel behaviour," Journal of Transport Geography, vol. 62, pp. 111-121, 2017. 
[5] S. C. Wirasinghe and N. S. Ghoneim, "Spacing of bus-stops for many to many travel demand," Transportation Science, vol. 15, no. 3, pp. 210-221, 1981.

[6] S. Mavoaa, K. Wittena, T. McCreanorb, and D. O’Sullivanc, "GIS based destination accessibility via public transit and walking in Auckland, New Zealand," Journal of transport geography, vol. 20, no. 1, pp. 15-22, 2012.

[7] F. Delgado, J. C. Munoz, and R. Giesen, "How much can holding and/or limiting boarding improve transit performance?" Transportation Research Part B: Methodological, vol. 46, no. 9, pp. 1202-1217, 2012.

[8] R. M. Stanger and V. R. Vuchic, "The design of bus-rail transit facilities," Transit Journal, vol. 5, pp. 61-72, 1979.

[9] J. A. Dunn Jr., "Coordination of urban transit services: The german model," Transportation, vol. 9, no. 1, pp. 33-43, 1980.

[10] A. D. May, "Integrated transport strategies: a new approach to urban transport policy formulation in the u.k.: foreign summaries," Transport Reviews, vol. 11, no. 3, pp. 223-247, 1991.

[11] L. Deng, W. Gao, Y. Fu, and W. Zhou, "Optimal design of the feeder-bus network based on the transfer system," Discrete Dynamics in Nature and Society, vol. 2013, Article ID 483682, pp. 1-10, 2013.

[12] B. F. Byrne and V. Vuchic, "Public transportation line positions and headways for minimum cost," in Traffic Flow and Transportation, pp. 347-360, 1972.

[13] V. F. Hurdle, "Minimum cost locations for parallel public transit lines," Transport Science, vol. 7, pp. 340-350, 1973.

[14] B. F. Byrne, "Cost minimizing positions, lengths and headways for parallel public transit lines having different speeds," Transportation Research, vol. 10, no. 3, pp. 209-214, 1976.

[15] S. Chien and Z. Yang, "Optimal feeder bus routes on irregular street networks," Journal of Advanced Transportation, vol. 34, no. 2, pp. 213-248, 2000.

[16] G. K. Kuah and J. Perl, "The feeder-bus network-design problem," Journal of the Operational Research Society, vol. 40, no. 8, pp. 751-767, 1989.

[17] C. L. Martins and M. V. Pato, "Search strategies for the feeder bus network design problem," European Journal of Operational Research, vol. 106, no. 2-3, pp. 425-440, 1998.

[18] A. S. Mohaymany and A. Gholami, "Multimodal feeder network design problem: ant colony optimization approach," Journal of Transportation Engineering, vol. 136, no. 4, pp. 323-331, 2010.

[19] L. M. Martínez and T. Eiró, "An Optimization Procedure to Design a Minibus Feeder Service: An Application to the Sintra Rail Line," Procedia - Social and Behavioral Sciences, vol. 54, pp. 525-536, 2012.

[20] S. N. Kuan, H. L. Ong, and K. M. Ng, "Solving the feeder bus network design problem by genetic algorithms and ant colony optimization," Advances in Engineering Software, vol. 37, no. 6, pp. 351-359, 2006.

[21] P. Shrivastava and M. O’Mahony, “A model for development of optimized feeder routes and coordinated schedules: a genetic algorithms approach," Transport Policy, vol. 13, no. 5, pp. 413425, 2006.

[22] P. Shrivastava and M. O’Mahony, “Design of feeder route network using combined genetic algorithm and specialized repair heuristic," Journal of Public Transportation, vol. 10, no. 2, 2007.

[23] P. Shrivastava and M. O’Mahony, "Use of a hybrid algorithm for modeling coordinated feeder bus route network at suburban railway station," Journal of Transportation Engineering, vol. 135, no. 1, pp. 1-8, 2009.
[24] A. Gholami and A. S. Mohaymany, "Economic conditions for minibus usage in a multimodal feeder network," Transportation Planning and Technology, vol. 34, no. 8, pp. 839-856, 2011.

[25] E. Cipriani, S. Gori, and M. Petrelli, “Transit network design: a procedure and an application to a large urban area," Transportation Research Part C: Emerging Technologies, vol. 20, no. 1, pp. 3-14, 2012.

[26] M. H. Almasi, A. Sadollah, S. M. Mounes, and M. R. Karim, "Optimization of a transit services model with a feeder bus and rail system using metaheuristic algorithms," Journal of Computing in Civil Engineering, vol. 29, no. 6, Article ID 04014090, 2015.

[27] S. Pan, J. Yu, X. Yang, Y. Liu, and N. Zou, "Designing a flexible feeder transit system serving irregularly shaped and gated communities: Determining service area and feeder route planning," Journal of Urban Planning and Development, vol. 141, no. 3, Article ID 04014028, 2015.

[28] G. K. Kuah and J. Perl, "Opyimization of feeder bus routes and bus-stop spacing," Journal of Transportation Engineering, vol. 114, no. 3, pp. 341-354, 1988.

[29] S. C. Wirasinghe, V. F. Hurdle, and G. Newell, "Optimal parameters for a coordinated rail and bus transit system," Transportation Science, vol. 11, no. 4, pp. 359-374, 1977.

[30] S. N. Kuan, H. L. Ong, and K. M. Ng, "Applying metaheuristics to feeder bus network design problem," Asia-Pacific Journal of Operational Research, vol. 21, no. 4, pp. 543-560, 2004.

[31] F. Zhao and X. G. Zeng, "Simulated annealing-genetic algorithm for transit network optimization," Journal of Computing in Civil Engineering, vol. 20, no. 1, pp. 57-68, 2006.

[32] C. R. Reeves, Ed., Modern Heuristic Techniques for Combinatorial Problems, John Wiley \& Sons, New York, NY, USA, 1993.

[33] S. M. Chowdhury and S. I.-J. Chien, "Intermodal transit system coordination," Transportation Planning and Technology, vol. 25, no. 4, pp. 257-287, 2002.

[34] A. Pradhan and G. Mahinthakumar, "Finding all-pairs shortest path for a large-scale transportation network using parallel Floyd-Warshall and parallel Dijkstra algorithms," Journal of Computing in Civil Engineering, vol. 27, no. 3, pp. 263-273, 2013.

[35] M. Hadi Baaj and H. S. Mahmassani, "Hybrid route generation heuristic algorithm for the design of transit networks," Transportation Research Part C: Emerging Technologies, vol. 3, no. 1, pp. 31-50, 1995.

[36] W. Fan and R. B. Machemehl, "Optimal transit route network design problem with variable transit demand: genetic algorithm approach," Journal of Transportation Engineering, vol. 132, no. 1, pp. 40-51, 2006.

[37] J.-F. Cordeau, M. Gendreau, G. Laporte, J.-Y. Potvin, and F. Semet, "A guide to vehicle routing heuristics," Journal of the Operational Research Society, vol. 53, no. 5, pp. 512-522, 2002.

[38] D. L. Miller, "A Matching Based Exact Algorithm for Capacitated Vehicle Routing Problems," ORSA Journal on Computing, vol. 7, no. 1, pp. 1-9, 1995. 


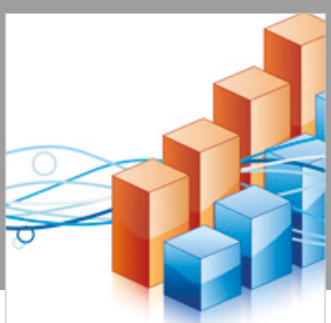

Advances in

Operations Research

\section{-n-m}
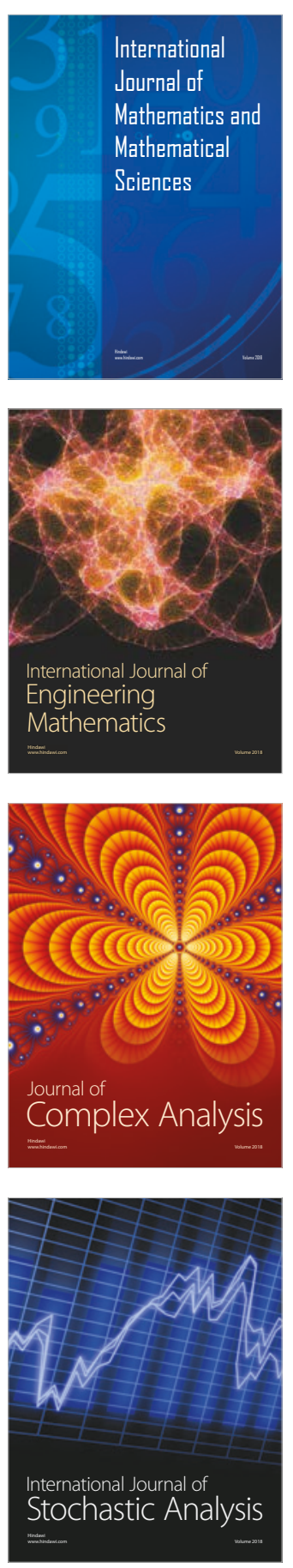
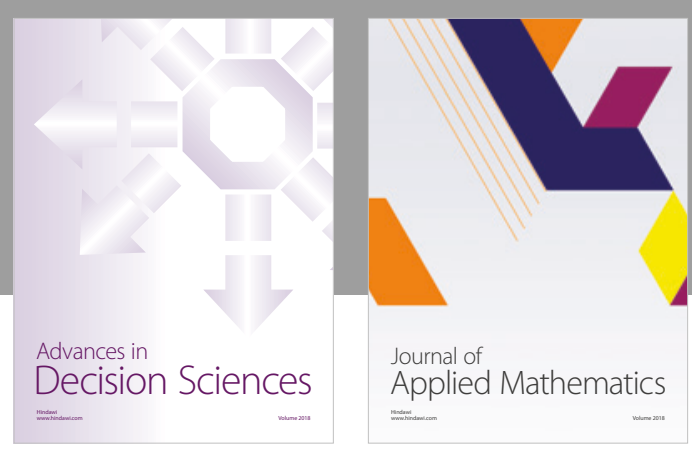

Journal of

Applied Mathematics
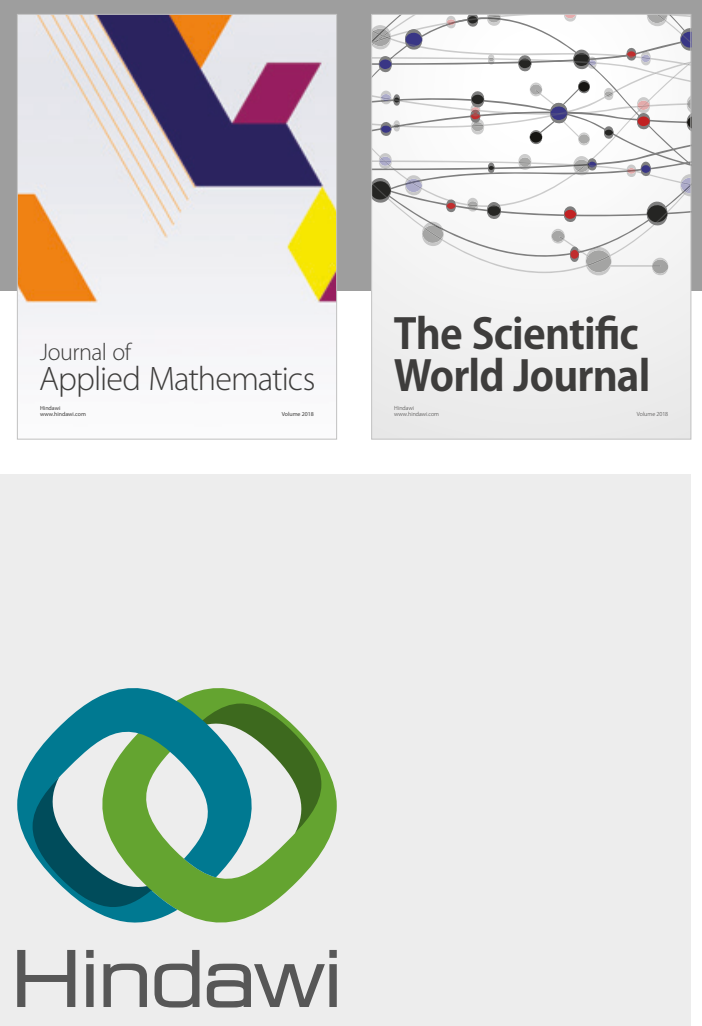

Submit your manuscripts at

www.hindawi.com

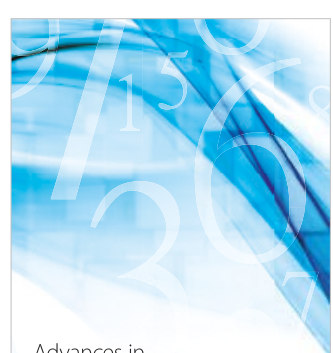

Advances in
Numerical Analysis
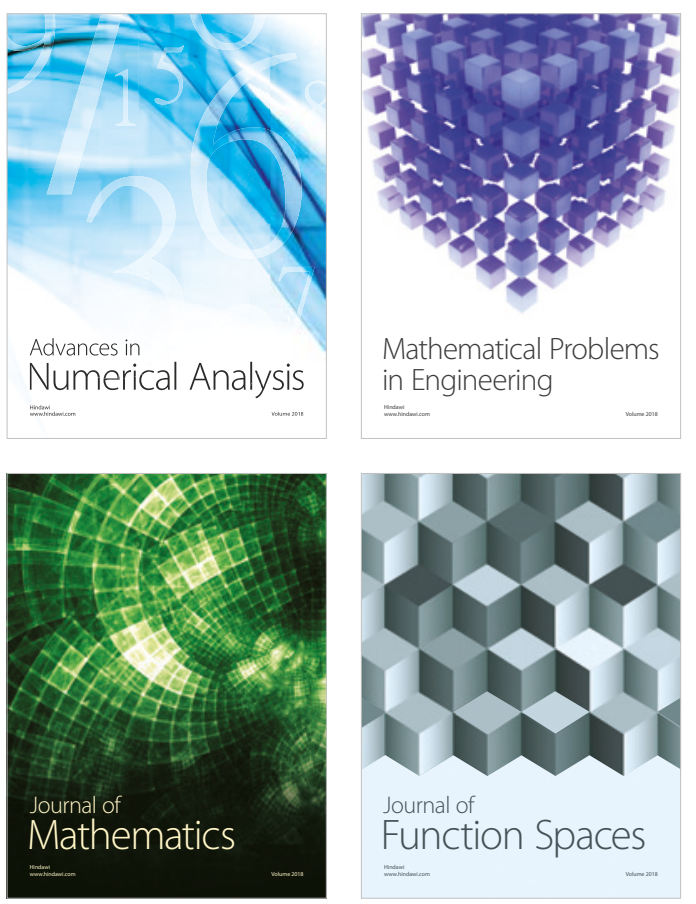

Mathematical Problems in Engineering

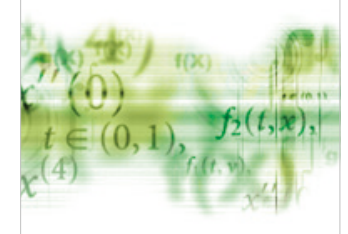

International Journal of

Differential Equations

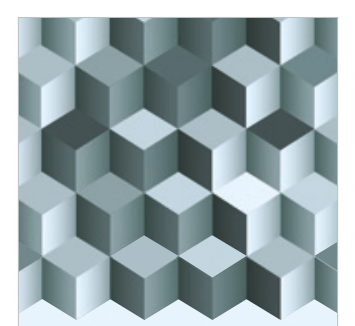

Journal of

Function Spaces

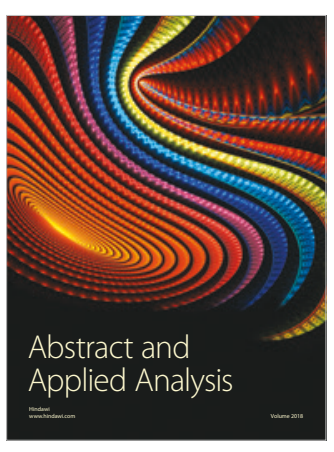

The Scientific

World Journal

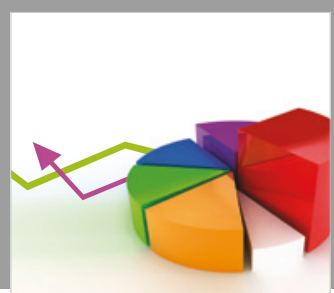

Journal of

Probability and Statistics
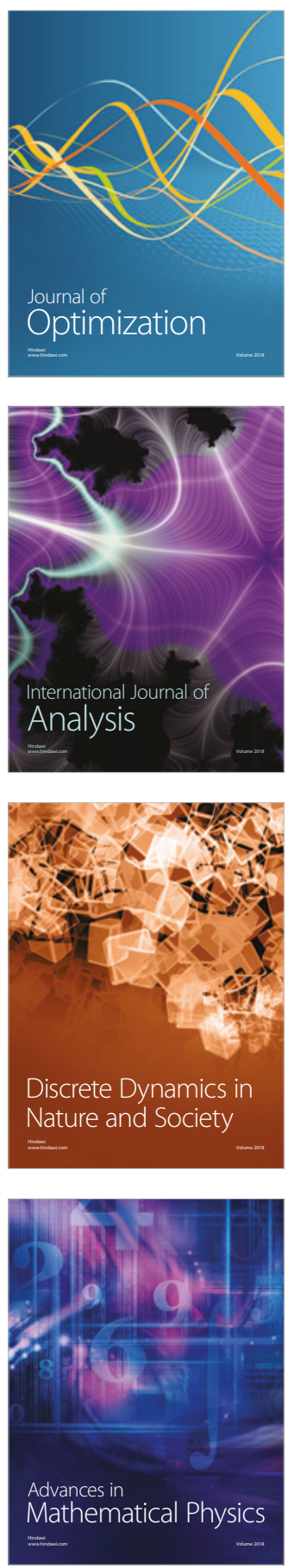